\title{
A Challenging Nonsurgical Management to Locally Advanced Urethral Squamous Cell Carcinoma
}

\author{
S Daoudi, S.H Touimi, I Adrif, A Lachgar, H Mrabti, N Benjaafar ,H Errihani
}

\begin{abstract}
Urethral squamous cell carcinoma (SCC) is a rare entity, comprising less than $1 \%$ of all cancers. The diagnosis is often late because the symptomatology is not specific. Its treatment is essentially surgical but often mutilating. The prognosis remains unfavorable in the majority of cases. The current hope is the combination of chemotherapy and radiotherapy.

We report a case of squamous cell carcinoma of the urethra which was discovered at a locally advanced stage and treated by neoadjuvant chemotherapy then concomitant chemoradiotherapy that showed complete response to this treatment.
\end{abstract}

Index Terms - Concomitant chemoradiotherapy, Locally advanced tumor, Neoadjuvant chemotherapy, Urethral squamous cell carcinoma

\section{INTRODUCTION}

Squamous cell carcinoma (SCC) of the urethra is rare, accounting for less than $1 \%$ of all cancers [1]. It occurs most often around the sixties [2]. The main predisposing factors are chronic inflammation, urethral stricture, radiotherapy, and urethritis in sexually transmitted diseases [3].

The diagnosis of these tumors is generally difficult because the symptomatology is not very specific and dominated by the presence of a palpable mass on the path of the urethra and the existence of an obstructive urinary syndrome [4].

Patients usually present with advanced stage disease and often have a poor outcome with a 5-year overall survival of approximately $40 \%$ [5]. Moreover, there are a limited number of randomized controlled trials to evaluate the optimal management of locally advanced urethral SCC because of its rarity [6].

Here, we present the successful use of the concomitant chemoradiation after traditional chemotherapy in neoadjuvant in a patient with locally advanced urethral squamous cell carcinoma (SCC).

\section{CASE REPORT}

A 35-year-old man presented with a 3-month history of

DAOUDI Sara, Department Of Medical Oncology, National Institute Of Oncology, Rabat, Morocco

TOUIMI Samia Hajar, Department Of Radiotherapy, National Institute Of Oncology, Rabat, Morocco

ADRIF Imane, Department Of Medical Oncology, National Institute Of Oncology, Rabat, Morocco difficulty urinating. Physical exam revealed an induration of the bulbar urethra with right inguinal lymphadenopathy. MRI of the penis showed a perineal tumor lesion extended to the spongy body and the membranous urethra measuring $35 \mathrm{~mm}$ in section and extending over a length of $80 \mathrm{~mm}$ to the prostate behind, the external sphincter of the anal canal behind and bottom and corpora cavernosa above, associated with right inguinal lymphadenopathy. A right inguinal lymph node dissection has been performed; the anatomopathological examination was in favor of a lymph node localization of a well-differentiated squamous cell carcinoma. A CT scan of chest, abdomen and pelvis and bone scintigraphy were negative for distant disease. Nevertheless, it was considered inoperable. In view of the extensive disease, the patient received three cycles of neoadjuvant therapy with 5-flurouracil and cisplatin with the intention of downstaging the tumor.
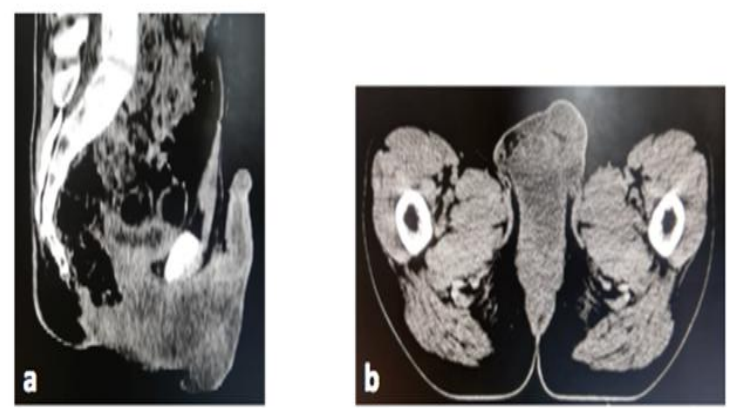

Figure 1: CT scan showing the urethral tumor before neoadjuvant chemotherapy a: Sagittal reconstruction b : Axial plan

Restaging imaging showed a $98 \%$ regression in tumor volume, confirming an excellent response to neoadjuvant chemotherapy. Afterward, the patient underwent concomitant chemoradiotherapy .The patient subsequently received perineal radiotherapy with Volumetric modulated arc therapy (VMAT). A dose of 66.6 Gy to the target volume (urethra), with a simultaneous dose of 45 Gy to the nodal volumes and 2 integrated boosts in 37 fractions of $1.8 \mathrm{~Gy}$ was planned, with concomitant weekly cisplatin chemotherapy at $40 \mathrm{mg} / \mathrm{m} 2$.We performed a pelvic MRI 2 months after the chemoradiation that shows a complete response with no residual tumor. 

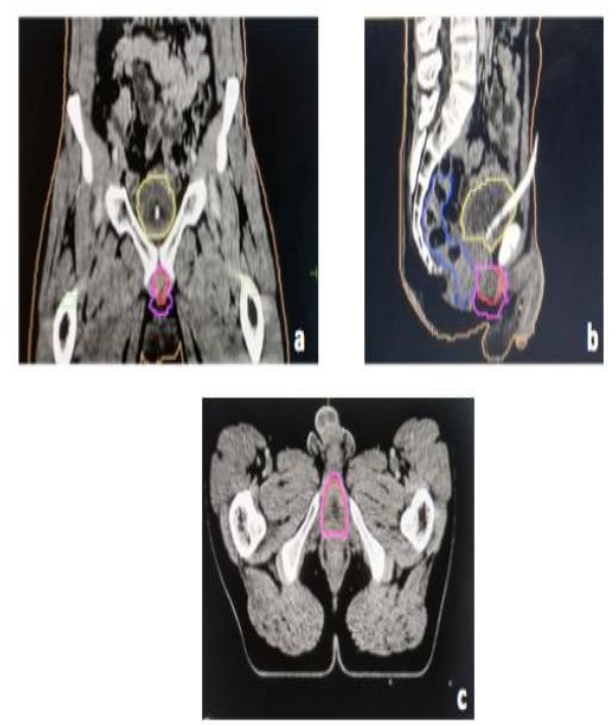

Figure 2: CT scan showing 98\% regression of the tumor after neoadjuvant chemotherapy a: Coronal reconstruction b: Sagittal reconstruction c: Axial plan

\section{DISCUSSION}

Urethral SCC is a rare malignancy and its management is particularly difficult due to the lack of evidence from randomized trials to inform practice. Multimodal therapy including chemotherapy, radiotherapy, and surgery is commonly used for invasive urethral SCC, unlike the management of SCC of other organs such as the vulva or anus, which can be managed nonsurgically [7].

Historically, urethral carcinoma was treated primarily with surgery, involving combinations of urethterectomy, partial penectomy, cystoprostatectomy with urinary diversion, and lymph node dissection [5]. It is important to note that surgery is often associated with complaints of reduction in the quality of life, reports of dissatisfaction of genital appearance, need for urinary diversion, and interference with daily activities [8]. Furthermore, treating patients with surgery alone has unfavorable results, with a treatment failure rate up to $75 \%$ [9].

Otherwise, Human papilloma virus (HPV) has been implicated in both male and female urethral cancers [1]. Wiener and colleagues found metastatic disease in $75 \%$ of HPV subtype 16-positive cases and also demonstrated a trend toward prolonged survival in HPV-positive cases compared with HPV-negative cases. Patients with HPV-positive urethral SCC tend to have better survival outcomes than HPV-negative cases [10].

The employment of radiotherapy alone in cases of bulbomembranous cancer resulted deleterious in several case reports [11], while combined use with chemotherapy showed positive results [12]. Kent et al reported on 29 men who were treated with chemoradiotherapy of 45 to 55 Gy plus 5-fluorouracil and mitomycin-C, which resulted in complete clinical response in $79 \%$ of patients [13]. Cohen et al analyzed 18 men who were treated with CRT, with 5-year overall and disease-free survival rates of $60 \%$ and $80 \%$, respectively [12].

Chemotherapy alone or concurrent chemoradiation can be used in the neoadjuvant setting, and cisplatin-containing drug regimens, in particular, have been shown to have good response [7].

Surgical consolidation and wide resection are still commonly used after neoadjuvant therapy [14]. Current National Comprehensive Cancer Network guidelines recommend chemoradiation for cT3/T4 and cN0-2 disease, with or without consolidative surgery [15].

In a series of 124 patients with clinically advanced tumor stage and/or node positive disease, Gakis et al demonstrated an advantage of neoadjuvant chemotherapy and neoadjuvant chemoradiotherapy over surgery alone or with adjuvant chemotherapy [16].

Nevertheless, with chemotherapy and radiation therapy, common toxicities such as cytopenia, skin necrosis, diarrhea, neuropathy, and sexual dysfunction can be seen.

In order to establish genital preservation for urethral SCC, Intensity modulated radiotherapy (IMRT) allows a more conformal dose distribution and may allow for dose escalation to the primary tumor with better sparing of organs at risk such as bladder and rectum [1].

Our experience suggests that using neoadjuvant chemotherapy to downstaging the tumor followed by chemoradiaotherapy, is highly effective in the treatment of locally advanced urethral SCC and that surgical consolidation can add little to no benefit with a high morbidity.

\section{CONCLUSION}

There is no consensus in literature about the most effective treatment for locally advanced SCC urethral. There may be an important role of nonsurgical management, which needs to be more studied. The current hope lies in the combinations of chemotherapy with radiotherapy.

\section{ACKNOWLEDGMENT}

We thank our oncologists and radiotherapists colleagues at national institute of oncology of Rabat who provided care and support for the patient. 


\section{REFERENCES}

[1] H. Coop, L. Pettit et al. Radical Chemoradiotherapy for Urethral Squamous Cell Carcinoma: Two Case Reports and a Review of the Literature. Hindawi. Case reports in Urology. Volume 2013, Article ID 194690, 4 pages.

[2] Navin Shah, Thomas Huebner, and Shannon Cherone. A Case of Squamous Cell Carcinoma of the Anterior Urethra in a Man. Rev Urol. 2019; 21(2-3): 133-135.

[3] Giuseppe Lucarelli, Marco Spilotros et al. A Challenging Surgical Approach to Locally Advanced Primary Urethral Carcinoma: A Case Report and Literature Review. Medicine (Baltimore). 2016 May; 95(19): e3642.

[4] Jilani Ghorbel, Ghassen Hafsia et al. Carcinome épidermoïde de l'urètre masculin révélé par une rupture spontanée de l'urètre. Can Urol Assoc J. 2011 Jun; 5(3): E36-E39.

[5] Tanmay Singh, Qing Kay Li and Daniel Y. Song. Pathologic Complete Response After Chemoradiation of a Massive Primary Urethral Carcinoma. Advances in Radiation Oncology (2019) 4, 487-491.

[6] Krishna H. Suthar, Meghana Kesireddy et al. Neoadjuvant Radiation with Concurrent 5-FU Resulting in Complete Pathologic Response in Stage IIIB Squamous Cell Carcinoma of the Urethra. Hindawi. Case Reports in Oncological Medicine. Volume 2020, Article ID 7948538, 3 pages.

[7] F. Dayyani, K. Hoffman, P. Eifel et al., "Management of advanced primary urethral carcinomas," BJU International, vol. 114, no. 1, pp. 25-31, 2014.

[8] N. Serrano, M. Chang, C. Leland Rogers et al., "Patient autonomy and shared decision making in the management of urethral cancer," Practical Radiation Oncology, vol. 6, no. 1, pp. 66-70, 2016.

[9] Serrano N, Chang M, Leland Rogers C, et al. Patient autonomy an shared decision making in the management of urethral cancer. Pract Radiat Oncol. 2016;6:66-70.

[10] Wiener JS, Effert PJ, Humphrey PA, et al. Prevalence of human papillomavirus types 16 and 18 in squamous-cell carcinoma of the penis: a retrospective analysis of primary and metastatic lesions by differential polymerase chain reaction. Int J Cancer. 1992;50:694-701.

[11] Zeidman EJ, Desmond P, Thompson IM. Surgical treatment of carcinoma of the male urethra. Urol Clin North Am 1992; 19:359-372.

[12] Cohen MS, Triaca V, Billmeyer B, et al. Coordinated chemoradiation therapy with genital preservation for the treatment of primary invasive carcinoma of the male urethra. J Urol 2008; 179:536-541.

[13] Kent M, Zinman L, Girshovich L, Sands J, Vanni A. Combined chemoradiation as primary treatment for invasive male urethral cancer. J Urol. 2015;193:532-537.

[14] C. P. Dinney, D. E. Johnson, D. A. Swanson, R. J. Babaian, and A. C. von Eschenbach, "Therapy and prognosis for male anterior urethral carcinoma: an update," Urology, vol. 43, no. 4,pp. 506-514, 1994.

[15] National Comprehensive Cancer Network. Bladder cancer, version 2.2018 . Available https://www.nccn.org/professionals/physician_gls/pdf/bladder.pdf. Accessed April 15, 2018.

[16] Gakis G, Morgan TM, Daneshmand S, et al. Impact of perioperative chemotherapy on survival in patients with advanced primary urethral cancer. Results of the international collaboration on primary urethral carcinoma. Ann Oncol 2015; 26:1754-1759. 